4. - On the properties of certain continued fractions, Proc. Amer. Math. Soc. vol. 3 (1952) pp. 921-937.

5. Oskar Perron, Die Lehre von den Kettenbrüchen, vol. II, Stuttgart, Teubner, 1957.

Technische Hochschule München,

University of California at Los ANGeles and

UNIVERSITY OF ILLINOIS

\title{
A THEOREM ON $A$-LOOPS
}

\section{J. MARSHALL OSBORN}

In a recent article, R. H. Bruck and Lowell J. Paige have investigated $A$-loops, or loops for which every element of the inner mapping group is an automorphism (see [1]). Specifically, they have shown that $A$-loops with the inverse property are diassociative and that there exist noncommutative diassociative $A$-loops. The authors also conjecture that the only commutative diassociative $A$-loops are the commutative Moufang loops. The purpose of the present note is to offer a proof of this conjecture.

Let $y$ and $z$ be two elements of a commutative diassociative $A$-loop $G$, and let $R_{y}$ denote right multiplication in $G$ by the element $y$. Then $S=R_{z} R_{y} R_{z y}^{-1}$ is an element of the inner mapping group, so that $w S \cdot x S=(w x) S$ for every pair of elements $w$ and $x$ of $G$. Setting $w=p q$ and $x=q^{-1}$ gives $(p q) S=p S \cdot\left[q^{-1} S\right]^{-1}$, and comparing with the first equation, we see that

$$
\begin{aligned}
x R_{z} R_{y} R_{z y}^{-1} & =x S=\left[x^{-1} S\right]^{-1}=\left[\left(x^{-1} z \cdot y\right) \cdot(z y)^{-1}\right]^{-1} \\
& =\left(x z^{-1} \cdot y^{-1}\right) \cdot(z y)=x R_{z}^{-1} R_{y}^{-1} R_{z y} .
\end{aligned}
$$

Thus ${ }^{1} R_{z} R_{y} R_{z y}^{-1}=R_{z}^{-1} R_{y}^{-1} R_{z y}$, or $R_{z y}^{2}=R_{y} R_{z}^{2} R_{y}$. Using diassociativity, we can write this as $\left(x y \cdot z^{2}\right) \cdot y=x \cdot\left(y \cdot z^{2} y\right)$, which is just a form of the Moufang identity except for the fact that $z$ is squared. Our problem is to show that the identity holds without this restriction.

We observe, first of all, that the subloop of $G$ consisting of all squares is a commutative Moufang loop. This already proves our theorem for loops all of whose elements have odd orders. One expects difficulty for loops containing elements of order two, particularly

Received by the editors October 12, 1957.

1 This formula may also be obtained from equation (3.24) of [1] by setting $L_{x}=R_{x}$. 
for loops containing only elements of order two, for which our weakened Moufang identity is vacuous. We begin by treating this special case.

Lemma 1. If $x, y$ are two elements of a commutative diassociative $A$ loop of exponent two, then:

(i) $R_{x} R_{y} R_{x y}=R_{x y} R_{x} R_{y}=R_{y} R_{x y} R_{x}$,

(ii) $\left(R_{x} R_{y} R_{x y}\right)^{2}=\left(R_{x} R_{y}\right)^{2}=\left(R_{y} R_{x}\right)^{2}=\left(R_{x y} R_{y}\right)^{2}$.

If $S=R_{x} R_{y} R_{x y}$, then $u S \cdot v S=(u v) S$, and setting $v=x y$ yields the first half of (i) immediately. The second half of (i) follows from this by replacing $x$ by $x y$ and $y$ by $x$. Also, if $S=R_{x} R_{y} R_{x} R_{y}$, then setting $v=x$ yields $\left(R_{x} R_{y}\right)^{2}=\left(R_{y} R_{x}\right)^{2}$, and using (i) and this relation gives all of (ii).

Lemma 2. Let $G$ be a commutative diassociative $A$-loop of exponent two, then $G$ is a group.

Let $a, b, c$ be three elements of $G$ and let $d=a b$ for convenience, then we compute: $c\left(R_{a} R_{b}\right)^{2}=c\left(R_{d} R_{b}\right)^{2}=[(c d \cdot b) \cdot d] \cdot b=d R_{c d \cdot b} R_{b}$ $=d R_{c d}\left(R_{c d} R_{c d \cdot b} R_{b}\right)=c R_{c d \cdot b} R_{b} R_{c d}=[c \cdot(c d \cdot b)] R_{b} R_{c d}=d\left(R_{c} R_{b}\right)^{2} R_{c d}$ $=d\left(R_{b} R_{c}\right)^{2} R_{c d}=a R_{c} R_{b} R_{c} R_{c d}=(a c \cdot a d) R_{c} R_{c d}=d R_{a} R_{a c} R_{c} R_{c d}$ $=d R_{c} R_{a} R_{a c} R_{c d}=[(c d \cdot a) \cdot a c] R_{c d}=c R_{a} R_{c d \cdot a} R_{c d}=c R_{c d} R_{a} R_{c d} \cdot a$ $=d R_{a} R_{c d \cdot a}=b \cdot(c d \cdot a)=c R_{d} R_{a} R_{b}=c R_{a b} R_{a} R_{b}$. Comparing the beginning and end of this computation yields $R_{a b}=R_{a} R_{b}$, so $G$ is associative.

Next, we consider the special case where the set of all squares forms a group. We need only consider loops with three generators, since the associative law involves only three elements, and for these it is sufficient to consider the free loop with the desired properties, since the others will be homomorphs of it.

Lemma 3. If $G$ is the free commutative diassociative $A$-loop on the generators $a, b, c$ with the property that the set of all squares forms a group, then $G$ is a group.

Let $x, y, z$ be three elements of $G$, then, using our weakened form of the Moufang identity, we compute: $z^{2} x \cdot y^{2}=\left(z^{2} x \cdot y^{2}\right) x \cdot x^{-1}$ $=\left(z^{2} \cdot y^{2} x^{2}\right) \cdot x^{-1}=\left(z^{2} y^{2} \cdot x^{2}\right) \cdot x^{-1}=z^{2} y^{2} \cdot x$. Hence,

$$
x y^{2} \cdot z=\left[\left(x z^{-1} \cdot z\right) \cdot y^{2}\right] \cdot z=x z^{-1} \cdot z^{2} y^{2}==\left(x z^{-1} \cdot z^{2}\right) \cdot y^{2}=x z \cdot y^{2},
$$

so that the square of any element of $G$ is in the center.

Now let $K$ be the group of all squares of elements of $G$, then $K$ is generated by $a^{2}, b^{2}$, and $c^{2}$, since $x \rightarrow x^{2}$ is an endomorphism. But $G$ 
has the free Abelian group on three generators as a homomorph, and the image of $K$ in this homomorph is a free Abelian group on the images of $a^{2}, b^{2}, c^{2}$. Hence, $K$ itself must be a free group. If $x, y, z$ are three elements of $G$ which do not associate, then $(x, y, z)$ has order two, since $(x, y, z)^{2}=\left[(x y \cdot z)\left(x^{-1} \cdot y^{-1} z^{-1}\right)\right]^{2}=\left(x^{2} y^{2} \cdot z^{2}\right)\left(x^{-2} \cdot y^{-2} z^{-2}\right)=1$. Then $(x, y, z)$ is not in $K$, so that the images of $x, y, z$ in $G / K$ will not associate. But $G / K$ is a group by Lemma 2, giving the desired contradiction.

We are now ready for the general case. Let $G$ be any commutative diassociative $A$-loop, and let $x, y, z$ be three arbitrary elements of $G$, then $\left(x^{2} y \cdot z^{6}\right) y=x^{2} \cdot z^{6} y^{2}=x^{2} z^{6} \cdot y^{2}=\left(x^{2} z^{6} \cdot y\right) \cdot y$, using the fact that the set of all squares forms a commutative Moufang loop, containing the sixth powers in its center. Thus, $y x \cdot z^{6}=\left(y x^{-1} \cdot x^{2}\right) \cdot z^{6}=y x^{-1} \cdot x^{2} z^{6}$ $=\left[\left(y x^{-1} \cdot x\right) \cdot z^{6}\right] \cdot x=y z^{6} \cdot x$, so sixth powers are in the center of $G$. Also, $x z^{2} \cdot y^{3}=\left[\left(x y^{-3} \cdot y^{3}\right) \cdot z^{2}\right] \cdot y^{3}=x y^{-3} \cdot y^{6} z^{2}=\left(x y^{-3} \cdot y^{6}\right) \cdot z^{2}=x y^{3} \cdot z^{2}$, so that any triple involving a cube and a square is associative. Now, the subloop $K$ of all cubes of $G$ forms a loop with the property that the subloop of all squares of elements in $K$ is a group, so $K$ itself must be a group by Lemma 3. Then $x^{3} y \cdot z^{3}=\left(x^{3} y \cdot z^{3}\right) y^{2} \cdot y^{-2}=\left(x^{3} y \cdot y^{2}\right) z^{3} \cdot y^{-2}$ $=\left(x^{3} y^{3} \cdot z^{3}\right) \cdot y^{-2}=\left(x^{3} z^{3} \cdot y^{3}\right) \cdot y^{-2}=x^{3} z^{3} \cdot y$. Finally, since $x^{2}$ and $x^{3}$ are both fixed under the automorphism $R_{y} R_{z^{3}} R_{y z^{3}}^{-1}$, so is $x^{3} x^{-2}=x$, proving that all cubes are in the center. The usual Moufang identity is now seen to hold, since $(x y \cdot z) y=z^{3} \cdot\left[\left(x y \cdot z^{-2}\right) \cdot y\right]=z^{3} \cdot\left[x \cdot\left(y \cdot z^{-2} y\right)\right]$ $=x \cdot(y \cdot z y)$. We have proved:

Theorem. Every commutative diassociative A-loop is also a commutative Moufang loop.

\section{BIBLIOGRAPHY}

1. R. H. Bruck and Lowell J. Paige, Loops whose inner mappings are automorphisms, Ann. of Math. vol. 63 (1956) pp. 308-323.

UNIVERSITY OF WISCONSIN 